\title{
Management of adrenocortical insufficiency with continuous subcutaneous hydrocortisone infusion: long-term experience in three patients
}

\author{
A Khanna', R Khurana², A Kyriacou ${ }^{3}$, R Davies ${ }^{2}$ and DW Ray ${ }^{1,2}$ \\ ${ }^{1}$ Faculty of Medical and Health Sciences, University of Manchester, Manchester M13 9PT, UK \\ ${ }^{2}$ Central Manchester University Hospitals NHS Foundation Trust, Manchester M13 9WL, UK \\ ${ }^{3}$ University Hospital of South Manchester, Manchester M23 9LT, UK
}

\author{
Correspondence \\ should be addressed \\ to D W Ray \\ Email \\ david.w.ray@manchester.ac.uk
}

\section{Summary}

To assess continuous subcutaneous hydrocortisone infusion (CSHI) in patients with adrenocortical insufficiency (Al) and difficulties with oral replacement. Three patients with $\mathrm{Al}$ and frequent hospital admissions attributed to adrenal crises were treated with CSHI, which was delivered via a continuous subcutaneous infusion. All three patients preferred CSHI and remained on it long term, which permitted prolonged follow-up analysis. All three patients reported symptomatic improvement, and in two cases, reduced hospital admission rates and inpatient stay lengths were observed. The cost of hospital admissions and overall treatment was reduced in all cases. CSHI offers a practical and acceptable alternative to oral replacement in a subset of patients with Al. The cost of initiating and maintaining the pump is offset in the long term by reduced frequency and duration of emergency admissions. CSHI can therefore be considered in a select group of patients who are resistant to treatment with conventional oral glucocorticoids.

\section{Learning points:}

- Continuous subcutaneous infusion of cortisol is a viable alternative in patients unable to take oral steroids.

- Patient acceptability was high, with three out of three patients preferring to remain on pump treatment.

- Hospital admissions were reduced in response to pump therapy, which compensated for the increased treatment cost.

- The daily dosage of hydrocortisone can be reduced by using pump therapy.

\section{Background}

The management of adrenocortical insufficiency (AI) conventionally involves oral glucocorticoid (GC) replacement therapy. Various regimes of oral GCs, ranging from multiple twice- or thrice-daily shorter-acting hydrocortisone (HC) to longer-acting preparations like prednisolone and dexamethasone, are commonly used. There are, however, a number of problems associated with oral GC therapies, which include first-pass hepatic metabolism and issues with oral delivery in gastrointestinal disease. In addition, oral therapy poorly mimics the normal circadian rhythm of cortisol secretion. Attempts to restore physiological diurnal variation in cortisol replacement with dual release $\mathrm{HC}$ has been shown to improve weight, blood pressure and glucose metabolism in patients with AI (1).

Poorly managed AI is associated with increased mortality (2) (3) and poorer quality of life (4). In a population-based study using a Swedish national hospital registry by Johannsson et al. (1), the all-cause mortality 
risk ratio was found to be more than twofold higher in primary AI, and this was attributed mainly to cardiovascular disease, cancers and infections. Possible explanations include over- (or under-) treatment with GCs or mineralocorticoids and associated autoimmune conditions (such as co-existent type 1 diabetes mellitus). DHEA deficiency in AI has also been proposed as a potential explanation for a lack of well-being in this patient group: some small studies have demonstrated benefit (5) (6) (7), whereas others have not (8).

It is very common in clinical practice for patients with AI to complain of fatigue and other symptoms during conventional treatment. An ideal GC replacement regime would be one that closely mimics the normal physiological cortisol profile. Continuous subcutaneous hydrocortisone infusion (CSHI), which has previously been shown to be well tolerated with lower total daily HC doses, aims to address this and other problems associated with conventional oral replacement therapy (9). There are a few potential problems that may be encountered with CSHI, namely, the cost associated with the apparatus used to deliver steroids and the use of an invasive subcutaneous cannula, which may predispose to local site infections, limit strenuous physical activity or dislodge and cause interruption of steroid delivery. However, CSHI was reported to be well tolerated in a previous study (9). We now report three cases of adrenal insufficiency managed successfully with CSHI. All were treated with CSHI on compassionate grounds, seeing as all three patients were poorly controlled on oral replacement therapy, and all suffered from upper gastrointestinal disease and vomiting, which both made oral administration difficult and also raised concerns in emergency staff about adrenal crisis, which resulted in frequent hospital admissions for parenteral GC therapy.

\section{Case presentation}

The first patient was a 53-year-old Caucasian female with a 6-year history of secondary adrenal suppression, which was attributed to a past medical history of long-term topical GC treatment for eczema. She had a percutaneous endoscopic gastrostomy for severe oesophageal dysmotility (nutcracker oesophagus). She also had poorly controlled epilepsy and a supra-pubic catheter in situ for atonic bladder. She presented frequently with recurrent urinary tract infections (UTI) and 'collapses' which were suggestive of seizures complicating hypoadrenal crises.

The second patient was a 50-year-old Caucasian female with adrenocorticotrophin (ACTH) deficiency of undetermined origin of 2 years duration. She had a background of severe gastroparesis and required feeding via a percutaneous endoscopic jejunostomy. She had renal impairment resulting from chronic pyelonephritis and

Table 1 Characteristics of the three patients at baseline and during established continuous subcutaneous cortisol infusion. TDD, total daily dose; 3DD, three divided doses. Lab reference values: 0900-1200 h (200-500 nmol/l), 0000-0900 h (50-250 nmol).

\begin{tabular}{|c|c|c|c|c|}
\hline Patient & $\begin{array}{l}\text { Starting rate of hydro- } \\
\text { cortisone }(\mathrm{mg} / \mathrm{h})\end{array}$ & $\begin{array}{l}\text { Mean serum cortisol } \\
(\mathrm{nmol} / / \mathrm{l})\end{array}$ & $\begin{array}{c}\text { Adjusted rate of } \\
\text { hydrocortisone }(\mathrm{mg} / \mathrm{h})\end{array}$ & $\begin{array}{c}\text { Subsequent mean } \\
\text { serum cortisol ( } \mathrm{nmol} / \mathrm{l})\end{array}$ \\
\hline \multicolumn{5}{|c|}{ Patient 1 (TDD $20 \mathrm{mg}$ in 3DD) } \\
\hline 0400-0900 & 1.1 & - & 1.1 & - \\
\hline 0900-1400 & 0.9 & 430 & 0.9 & 357 \\
\hline $1400-1800$ & 0.8 & 385 & 0.8 & 272 \\
\hline $1800-2200$ & 0.6 & 344 & 0.6 & 251 \\
\hline 2200-0400 & 0.7 & - & 0.6 & - \\
\hline Total over 24 h (mg) & 19.8 & - & 19.2 & - \\
\hline \multicolumn{5}{|c|}{ Patient 2 (TDD $30 \mathrm{mg}$ in 3DD) } \\
\hline 0400-0900 & 1 & - & 1.3 & - \\
\hline 0900-1400 & 0.7 & - & 1 & - \\
\hline $1400-1800$ & 0.6 & - & 0.9 & - \\
\hline $1800-2200$ & 0.4 & - & 0.7 & - \\
\hline $2200-0400$ & 0.5 & - & 0.8 & - \\
\hline Total over 24 h (mg) & 15.5 & - & 23.6 & - \\
\hline \multicolumn{5}{|c|}{ Patient 3 (TDD $20 \mathrm{mg}$ in 3DD) } \\
\hline 0400-0900 & 1 & - & 1 & - \\
\hline $0900-1400$ & 0.7 & 400 & 0.8 & - \\
\hline $1400-1800$ & 0.6 & 292 & 0.7 & - \\
\hline $1800-2200$ & 0.4 & 163 & 0.5 & - \\
\hline $2200-0400$ & 0.5 & 91 & 0.6 & - \\
\hline Total over 24 h (mg) & 15.5 & - & 17.4 & - \\
\hline
\end{tabular}




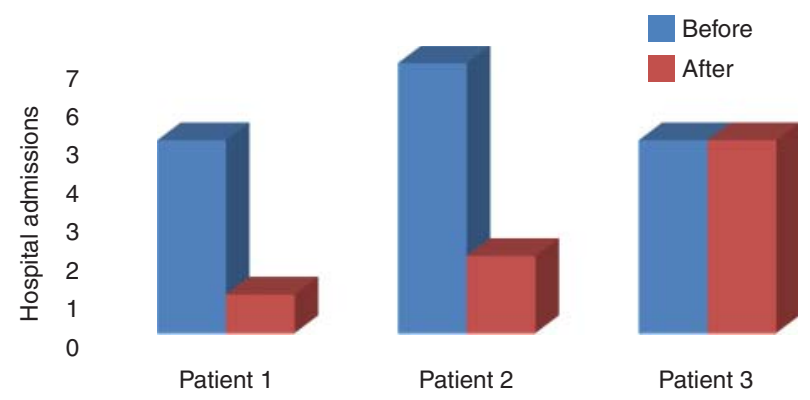

Figure 1

The number of hospital admissions during the 12 months before and 12 months after the introduction of CSHI therapy.

required frequent hospital admissions because of UTI episodes that resulted in acute on chronic deterioration of her renal function.

The third patient was a 33-year-old Caucasian female with secondary adrenal suppression resulting from longterm topical and intramuscular GCs for severe atopic dermatitis. Her other co-morbidities were irritable bowel syndrome, migraines and a complex psychological background that included severe depression. She had difficulties maintaining regular multiple $\mathrm{HC}$ dosing because she often forgot to take medications.

All three of the present patients were considered for CSHI because of their poor quality of life as well as their numerous and frequently prolonged emergency admissions for symptoms ranging from mild nausea and vomiting to frank Addisonian crises. In many cases, emergency admissions out of hours were poorly documented, and the role of steroid deficiency was not clear. Despite this, all three were subject to frequent high-dose parenteral GC administration. Additional management complications included the unreliability of absorption via the oral route (patients 1 and 2) and marked psychological factors and compliance issues (patient 3).

\section{Investigation}

All three patients were commenced on CSHI with Efcortisol $(100 \mathrm{mg} / \mathrm{ml}$, Sovereign Medical, Stansted, Essex, UK) delivered via a continuous subcutaneous insulin infusion (CSII) 554 Medtronic Pump (Watford, UK). Infusion rates were considered in blocks, with the 0400-0900 $\mathrm{h}$ morning period being the peak infusion time, as in normal physiology. No formal calculation was used to determine the dosing of cortisol. The previous total daily oral dose of HC, the patient's body weight, and discussions with the patient were used to determine the average hourly infusion rate. The hourly infusion rate was then multiplied by an arbitrary value for the morning period (1.3 for the first patient and 1.5 for the other two patients). The doses were then gradually reduced slowly throughout the day. All of our patients received intensive education on self-managing pumps and caring for cannula sites in addition to reinforced education regarding sickday rules. The pumps were also set up with a temporary basal rate, which was double the usual rate of use during periods of stress and minor illness. They were also provided with an emergency supply of intramuscular HC.

All three of our patients were monitored closely during the first week after commencement with telephone contact and regular in-person reviews for clinical assessment. Patients were encouraged to provide samples for biochemical monitoring via either serum or salivary cortisol levels to enable the titration of HC infusion rates. ACTH was not measured because it was already known to be deficient in all three cases. In addition, ACTH is highly labile and not useful as a biomarker of the hypothalamic-pituitary-adrenal axis.

The first patient to start CSHI was on a total of $20 \mathrm{mg}$ daily of HC, and the initial rate was started at equivalent dosage (Table 1). This was reduced after biochemical monitoring with serum cortisol day profile, and a subsequent serum cortisol day profile was satisfactory (10).

We initiated our second patient on CSHI a few months later, and in her case, the initial total daily dose was about $50 \%$ of her previous daily oral HC dose (Table 1). This was based on clinical judgement in view of her weight and not on any formal calculation. The infusion rates were titrated based on clinical symptoms and clinical judgement, seeing as it has not yet been possible to perform a cortisol profile because of patient preference. Her total daily dose remains less than the previous total oral HC dose.

Our third patient to start CSHI was commenced on $75 \%$ of her total daily oral HC dose (Table 1). Her serum cortisol levels were satisfactory during initial monitoring,

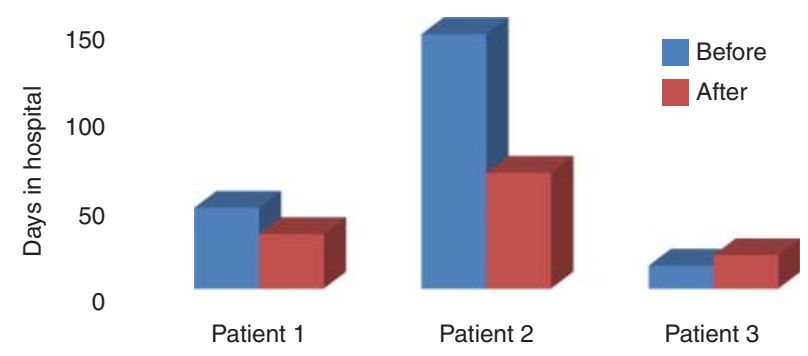

Figure 2

The number of days spent in hospital during the 12 months before and 12 months after the introduction of CSHI therapy. 
Table 2 The estimated costs of care for each patient. Hydrocortisone tablet costs were estimated using the patient prescriptions and prices indicated in the British National Formulary (BNF) 64 (2012). The costs of the CSHI pump and consumables were calculated by the pricings charged to the hospital trust. Admission costs were estimated using NHS admissions Healthcare Resource Group (HRG) codes.

\begin{tabular}{|c|c|c|}
\hline & $\begin{array}{l}\text { Estimated cost of } \\
\text { admissions and } \\
\text { hospital treatment } \\
\text { in the } 12 \text { months } \\
\text { before CSHI }\end{array}$ & $\begin{array}{c}\text { Annual cost of } \\
\text { hydrocortisone } \\
\text { tablets }\end{array}$ \\
\hline Patient 1 & f11 223 & f1207 \\
\hline Patient 2 & f17 668 & f1225 \\
\hline Patient 3 & $f 6872$ & £1207 \\
\hline
\end{tabular}

but the infusion rates were adjusted once again based on her clinical response, such as her subjective perception of energy levels. Unfortunately, it was not been possible to obtain further samples for continued monitoring.

\section{Treatment}

CSHI with Efcortisol (100 mg/ml, Sovereign) delivered via a CSII 554 Medtronic pump. Funding for the pumps was provided by the Wellcome Trust, although the costs of the consumables were covered by the NHS.

\section{Outcome and follow-up}

The average duration of CSHI for our patients at this time is 17.3 months (20, 17 and 15 months). All three patients were encouraged to discuss the impact of CSHI and their perceived changes in their quality of life during their 6-month follow-up clinic appointments. All three patients reported a significant improvement in overall well-being on CSHI and would choose it indefinitely as their preferred mode of HC replacement therapy. Besides this subjective improvement, in two patients, the number and duration of emergency admissions within the 12 months after intervention was also reduced as compared to the 12-month period before using CSHI (Figs 1 and 2). One of our patients also successfully managed periods of intercurrent illness with UTI by temporarily increasing the basal rate for $48 \mathrm{~h}$ during these episodes, and she thus avoided emergency admissions that would have been inevitable on oral replacement.

The total daily dose of HC delivered via CSHI was also on average $13.85 \%$ less than the previous total oral doses of HC had been $(7.25,21.3$ and 13\% less in the in first, second and third cases respectively).

Local site infections were not in seen our patient group; they are uncommon with CSII, and it remains to be seen whether subcutaneous HC carries a different risk of localised infections at the infusion site as compared to insulin infusion.

One of the main disadvantages of CSHI is, of course, that the cost of initiating and maintaining the pump is significantly greater than that of oral GC treatment. CSHI can, however, reduce recurrent and lengthy admissions, as was seen in our patients, and in the long term, it has proven cost effective when compared with the cost of impatient admission (Table 2, Fig. 3).

\section{Discussion}

Our intervention in three patients in a real-life clinical care setting demonstrates the efficacy of CSHI in greatly improving symptoms and quality of life. We also successfully reduced hospital admission rates and lengths in two of our three patients. In the case of the third patient, hospital admission rates remained the same, and the length of inpatient stay increased. The costs associated with hospital admissions and the overall treatment burden had, however, been reduced significantly. These costs were calculated using patient prescriptions and the 64th edition of the British National Formulary (BNF) in 2012 as well as the prices of CSHI pumps and consumables

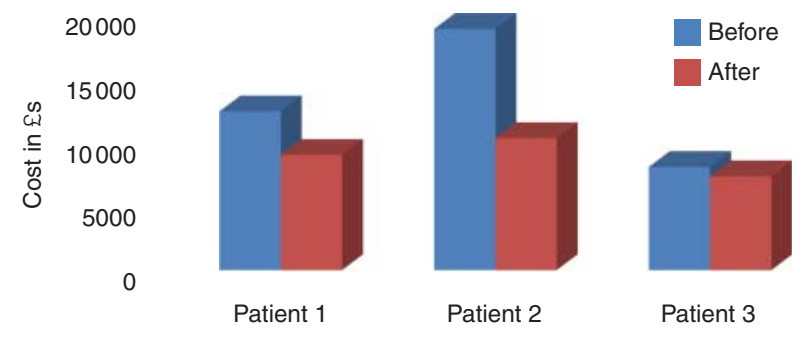

Figure 3

The cost related to hospital admissions and maintenance treatment during the 12 months before and 12 months after introduction of CSHI therapy. 
as charged to the hospital trust and admission costs, which were estimated using the NHS admission health resource group codes.

Upon closer examination of these results, several observations can be made. First, the third patient required hospital admission with prolonged psychiatric problems that were unrelated to her adrenal disease. Furthermore, in a number of her hospital admissions, the acute medical admission did not have a clear diagnosis; it was therefore difficult to attribute many of them to adrenal disease. Second, she did not require any additional exogenous steroids during her subsequent admissions, which thereby reduced the inpatient costs. Finally, the patient's issues with adherence to medication were resolved with the pump.

Regarding the first two patients, there were clear benefits not only in terms of improved well-being, reduced hospital admissions and reduced inpatient stay length but also in terms of diagnostic clarification. Before the present intervention, there had been diagnostic uncertainty regarding the second patient's gastrointestinal symptoms, with the concern that her nausea and vomiting may be the result of a hypoadrenal state. This resulted in treatment with intravenous steroids and inpatient monitoring. After CSHI, however, because her serum cortisol was known to be within the normal range and administration could be readily titrated in response to acute illness, there was less diagnostic uncertainty, and unnecessary treatment with intravenous GCs was avoided.

Because these three patients were treated pragmatically and on a compassionate basis, we did not formally assess quality of life before and after intervention. However, all three patients reported that their lives were transformed by the intervention, and all prefer to remain on the pump therapy despite the inconvenience associated with it. This was granted based on our findings.

No adverse effects were encountered by any of the patients from use of the pumps. Our intention with the application of CSHI was primarily to alleviate symptoms, and we achieved this successfully. Monitoring in two of the three patients demonstrated cortisol levels within normal physiological ranges throughout the day (10). This provided further evidence for the role of CSHI in maintaining normal circadian variation of cortisol levels, and it also positively correlated with our intended clinical outcomes.

The reduction in the frequency and length of hospital admissions is an important outcome for patients because it dramatically improves quality of life. Additionally, the reduction in cost to NHS trusts with CSHI is significant, and it can justify the use of expensive pumps in selected patients with poorly controlled AD. The costs of lengthy hospital admissions, inpatient management and oral HC treatment together exceed the costs associated with CSHI treatment. We propose that CSHI is a viable, practical treatment option in patients that require adrenal replacement therapy in whom conventional oral administration is unreliable or ineffective.

\section{Declaration of interest}

The authors declare that there is no conflict of interest that could be perceived as prejudicing the impartiality of the research reported.

\section{Funding}

This study was supported by the Wellcome Trust (to DWR).

\section{Patient consent}

Written consent has been obtained from all three patients.

\section{Author contribution statement}

A Khanna selected the patients, provided patient care and monitoring, obtained data, performed the analyses and wrote the paper; R Khurana obtained the patient consent and provided patient care and monitoring; A Kyriacou wrote the paper; $\mathrm{R}$ Davies provided patient care and instituted and adjusted the CSHI regimen; and DW Ray provided patient care, wrote the paper, and directed the study.

\section{References}

1 Johannsson G, Nilsson AG, Bergthorsdottir R, Burman P, Dahlqvist P, Ekman B, Engstrom BE, Olsson T, Ragnarsson O, Ryberg M et al. 2012 Improved cortisol exposure-time profile and outcome in patients with adrenal insufficiency: a prospective randomized trial of a novel hydrocortisone dual-release formulation. Journal of Clinical Endocrinology and Metabolism 97 473-481. (doi:10.1210/jc.2011-1926)

2 Bergthorsdottir R, Leonsson-Zachrisson M, Oden A \& Johannsson G 2006 Premature mortality in patients with Addison's disease: a population-based study. Journal of Clinical Endocrinology and Metabolism 91 4849-4853. (doi:10.1210/jc.2006-0076)

3 Bensing S, Brandt L, Tabaroj F, Sjoberg O, Nilsson B, Ekbom A, Blomqvist P \& Kampe O 2008 Increased death risk and altered cancer incidence pattern in patients with isolated or combined autoimmune primary adrenocortical insufficiency. Clinical Endocrinology 69 697-704. (doi:10.1111/j.1365-2265.2008.03340.x)

4 Bleicken B, Hahner S, Loeffler M, Ventz M, Allolio B \& Quinkler M 2008 Impaired subjective health status in chronic adrenal insufficiency: impact of different glucocorticoid replacement regimens. European Journal of Endocrinology 159 811-817. (doi:10.1530/EJE-08-0578)

5 Gurnell EM, Hunt PJ, Curran SE, Conway CL, Pullenayegum EM, Huppert FA, Compston JE, Herbert J \& Chatterjee VK 2008 Long-term DHEA, replacement in primary adrenal insufficiency: a randomized, controlled trial. Journal of Clinical Endocrinology and Metabolism 93 400-409. (doi:10.1210/jc.2007-1134)

6 Hunt PJ, Gurnell EM, Huppert FA, Richards C, Prevost AT, Wass JA, Herbert J \& Chatterjee VK 2000 Improvement in mood and fatigue after dehydroepiandrosterone replacement in Addison's disease in a 
Endocrinology

Diabetes \& Metabolism

CASE REPORT
A Khanna and others
Management of adrenocortical insufficiency
ID: 15-0005; May 2015

DOI: 10.1530/EDM-15-0005 randomized, double blind trial. Journal of Clinical Endocrinology and Metabolism 85 4650-4656. (doi:10.1210/jcem.85.12.7022)

7 Arlt W, Callies F, van Vlijmen JC, Koehler I, Reincke M, Bidlingmaier M, Huebler D, Oettel M, Ernst M, Schulte HM et al. 1999 Dehydroepiandrosterone replacement in women with adrenal insufficiency. New England Journal of Medicine 341 1013-1020. (doi:10.1056/ NEJM199909303411401)

8 Lovas K, Gebre-Medhin G, Trovik TS, Fougner KJ, Uhlving S, Nedrebo BG, Myking OL, Kampe O \& Husebye ES 2003 Replacement of dehydroepiandrosterone in adrenal failure: no benefit for subjective health status and sexuality in a 9-month, randomized, parallel group clinical trial. Journal of Clinical Endocrinology and Metabolism $\mathbf{8 8}$ 1112-1118. (doi:10.1210/jc.2002-020769)

9 Lovas K \& Husebye ES 2007 Continuous subcutaneous hydrocortisone infusion in Addison's disease. European Journal of Endocrinology 157 109-112. (doi:10.1530/EJE-07-0052)

10 Van Cauter E, Leproult R \& Kupfer DJ 1996 Effects of gender and age on the levels and circadian rhythmicity of plasma cortisol. Journal of Clinical Endocrinology and Metabolism 81 2468-2473. (doi:10.1210/jc. 81.7.2468)

Received in final form 13 March 2015

Accepted 27 April 2015 